\title{
Filigrane
}

Écoutes psychothérapiques

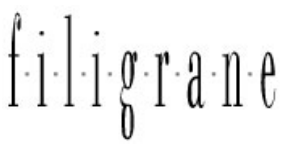

\section{L'après-coup, un bain de jouvence pour la psyché}

\section{Bernard Chervet}

Volume 22, numéro 2, automne 2013

Psychanalyse et temporalités II

URI : https://id.erudit.org/iderudit/1022555ar

DOI : https://doi.org/10.7202/1022555ar

Aller au sommaire du numéro

Éditeur(s)

Revue Santé mentale au Québec

ISSN

1192-1412 (imprimé)

1911-4656 (numérique)

Découvrir la revue

Citer cet article

Chervet, B. (2013). L'après-coup, un bain de jouvence pour la psyché. Filigrane, 22(2), 33-54. https://doi.org/10.7202/1022555ar

\section{Résumé de l'article}

Une étude du procès de l'après-coup permet de discerner les temporalités qui coexistent au sein du psychisme. L'auteur propose d'examiner celles-ci à partir d'une séquence clinique qu'il confronte ensuite au processus de théorisation de Freud. Il replace ainsi le concept d'après-coup dans l'histoire de la métapsychologie. Sont rencontrées la régression temporelle, l'intemporalité, l'atemporalité du traumatique, ainsi que les opérations de substitution et de résolution, créatrices du passé, de l'avenir et du présent. En introduisant l'intemporalité des désirs inconscients, la doctrine du rêve nous renseigne sur le travail psychique en deux temps exigé par l'existence de l'atemporalité traumatique, ainsi que sur les capacités de régénérescence libidinale du rêve. Celui-ci s'avère remplir la fonction de bain de jouvence. La méthode psychanalytique reprend à son compte le travail très original de l'entre-deux-temps qu'est le travail de rêve, et l'inscrit en séance en tant que travail de transfert, afin de promouvoir l'effet thérapeutique escompté. 


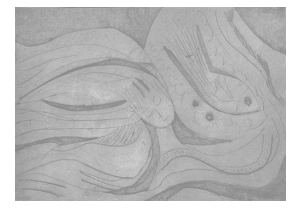

\section{L'après-coup, un bain de jouvence pour la psyché}

\section{Bernard Chervet}

Une étude du procès de l'après-coup permet de discerner les temporalités qui coexistent au sein du psychisme. L'auteur propose d'examiner celles-ci à partir d'une séquence clinique qu'il confronte ensuite au processus de théorisation de Freud. II replace ainsi le concept d'après-coup dans I'histoire de la métapsychologie. Sont rencontrées la régression temporelle, I'intemporalité, I'atemporalité du traumatique, ainsi que les opérations de substitution et de résolution, créatrices du passé, de l'avenir et du présent. En introduisant l'intemporalité des désirs inconscients, la doctrine du rêve nous renseigne sur le travail psychique en deux temps exigé par l'existence de l'atemporalité traumatique, ainsi que sur les capacités de régénérescence libidinale du rêve. Celui-ci s'avère remplir la fonction de bain de jouvence. La méthode psychanalytique reprend à son compte le travail très original de l'entre-deux-temps qu'est le travail de rêve, et l'inscrit en séance en tant que travail de transfert, afin de promouvoir l'effet thérapeutique escompté.

'après-coup est un concept psychanalytique qui permet tout particuliè- rement une réflexion sur le temps et sur les temporalités spécifiques de la vie psychique. Ce terme désigne un procès psychique complexe impliquant plusieurs temporalités imbriquées.

Il convient d'office de bien différencier le substantif après-coup, Nachträglichkeit de l'adjectif et adverbe après coup, Nachträglich. Le signifié immédiat de ce dernier reste proche des aperceptions empiristes du temps, depuis l'élection d'un point de départ, un moment dit temps zéro, la mesure d'une durée décrivant une période, jusqu'à l'inexorable flèche du temps ou l'éternel retour d'un temps circulaire.

\section{Le cheminement historique du concept d'après-coup}

Le substantif féminin allemand Nachträglichkeit est un néologisme fabriqué par Freud en 1897 (cf. la lettre à Fließ no 146) à partir du terme Nachträglich de la langue allemande courante. Toutefois, l'idée était déjà clairement formulée deux ans plus tôt (Freud, 1895). La traduction française a 
fait de même. Elle a néologisé un terme courant de la langue, et a placé un tiret dans l'orthographe du nom et concept après-coup, alors que l'adjectif et l'adverbe de la langue commune n'en ont traditionnellement pas.

Il n'existe que onze occurrences dans l'œuvre de Freud, correspondance incluse, d'utilisation de ce substantif. La particularité temporelle de l'usage par Freud de ce terme mérite d'être soulignée. Freud cesse en effet de l'utiliser de façon manifeste en 1917, bien qu'il continue ensuite à se référer de façon très déterminée au procès psychique en deux temps qu'il désigne.

En France, c'est Lacan qui en fut le promoteur. Il eut recours à ce concept pour régénérer la psychanalyse des années 1950 afin de la ré-ouvrir à l'esprit freudien, jugeant, en partie avec raison, qu'elle l'avait perdu, avec le risque de dégradation en psychologie du moi. L'appel de Lacan à ce «retour à Freud» se situe dans un contexte temporel précis, celui d'un processus de deuil interrompu et en suspens. En effet, la mort de Freud en 1939, avait été quelque peu obérée par la seconde guerre mondiale. Cet appel constitue donc en lui-même, un après-coup de la valeur traumatique du décès de Freud pour la communauté analytique. Lacan s'identifie alors à cet appel au point de prendre comme identité, d'incarner l'après-coup de Freud. Il se propose comme sa réactualisation, sa voix, sa résurrection, son exaltation.

Il embrase le concept de l'après-coup grâce à de très belles formules. Il rappelle que le «Nachträglich ou après-coup, selon lequel le trauma s'implique dans le symptôme, montre une structure temporelle d'un ordre plus élevé [que la rétroaction]» (Lacan, 1966). Se référant aux deux temps et à la mise en latence, il écrit: "L'après faisait antichambre, pour que l'avant pût prendre rang.» Il rehausse ainsi la causalité de la surdétermination inconsciente, et renoue avec la grande tradition psychiatrique française et parisienne, en particulier avec Charcot et sa description d'une séméiologie des troubles hystériques en deux temps: un temps 1 , celui du choc, et un temps 2 , celui du symptôme. Le génie séméiologique de Charcot lui a fait privilégier la dimension temporelle en décrivant un processus en deux temps, puis en dénommant l'entre-deux-temps, temps d'incubation psychique, ou d'élaboration psychique.

Lacan préfère décrire un processus en trois temps, composé de l'instant de voir, du temps de comprendre, et du moment de conclure (Lacan, 1966, 197213). Le premier temps vient mettre l'accent sur le « voir», justement là où le trauma laisse plutôt entendre qu'il n'y a rien à voir. Le second temps, celui du comprendre (prendre avec, en soi, à l'intérieur) rejoint l'élaboration psychique de l'entre-deux-temps. Mais l'accent porté sur la compréhension risque de faire passer à côté de la spécificité de la mise en latence et de la 
régression indispensable à l'avènement d'une future formation conclusive, ne serait-ce qu'un symptôme, ayant puisé son économie libidinale dans l'économie pulsionnelle régressive. Lacan ne reprend donc pas à son compte, à la différence de Freud, le modèle de la rétrogression de Breuer, ce processus de retour dans le temps, de réactualisation du passé au présent permettant une catharsis libératrice d'un désir, donc d'un avenir. De cette rétrogression nâ̂tra la régression temporelle de Freud, puis les régressions topique et libidinale, mais aussi la téléologie freudienne portée par la règle fondamentale des traitements psychanalytiques, l'orientation soutenue des investissements psychiques vers la conscience et le monde des objets.

\section{Approche métapsychologique du procès d'après-coup}

L'après-coup articule ces différentes étapes que sont la régression, la mutation et la production, selon une finalité dernière mais aussi première, consistant à promouvoir une prime de désir qui porte le nom d'avenir. Ce procès inclut donc une remontée dans le temps, une intemporalité conservatrice certes, mais surtout une régénération libidinale ayant valeur de rajeunissement.

Le prototype du procès de l'après-coup est l'activité de rêver, qui aboutit quand elle réussit à ce sentiment de régénérescence. Du point de vue économique, le sommeil-rêve est un bain de jouvence qui participe à une générativité, celle de la libido. Le sujet se réveille subjectivement, mais aussi libidinalement rajeuni.

Cette capacité si précieuse, idéalisée dans le célèbre at integrum, est annoncée en partie par les signifiés transmis par le terme lui-même. En allemand, Nachträglichkeit signifie "porter après », un "après » qui désigne un «vers l'avant»; donc "porter vers l'avant». Le double sens des mots s'impose et insiste: l'avant du passé, de ce qui est derrière, et le vers l'avant, vers ce qui est devant. Cette insistance du double sens participe à l'érotisation si aisée du terme. En français la dimension masochique impliquée dans le travail de l'après-coup se fait entendre encore plus nettement qu'en allemand. Les représentations de porter sont remplacées par les concaténations associatives liées à coup (Chervet, 2009, 1361-1441), ouvrant tout autant vers le sadomasochisme que vers le meurtre, et par l'argot vers l'acte sexuel.

\section{Les temporalités du travail de transfert}

Pour illustrer et complexifier ces propos introductifs, historiques et métapsychologiques, nous allons nous appuyer sur notre expérience quotidienne de la cure et des traitements analytiques, tant du point de vue de 
l'analyste que de l'analysant. En fait, toute la clinique des séances pourrait venir illustrer notre propos. Dans un travail conséquent sur l'après-coup, j'avais présenté 13 vignettes cliniques (Chervet, 2009, 1361-1441). Avec l'appel à la clinique, nous nous situons entre illustration-démonstration et complexification, chaque situation clinique apportant plus de nuances que le schématisme théorique néanmoins obligatoire pour que la clinique ait accès à l'intelligibilité. Nous retrouvons ici la formule bien connue de la réalité qui verdoie à jamais alors que la théorie n'est que grise. En psychanalyse, clinique et théorie ne peuvent être séparées de façon radicale. Tout récit clinique est organisé par une théorie plus ou moins implicite, et la théorie est elle-même un matériel clinique. La théorisation est une des composantes de la pensée humaine, preuve en sont les théories sexuelles infantiles. Ce qui n'est pas représentable par des contenus issus directement de traces perceptives et mnésiques, appelle et s'exprime par d'autres voies que les représentations, par la sensualité, les sentiments et les théories.

Un exemple. Un patient arrive à sa séance en évoquant un petit retard, et le fait qu'il a heurté deux personnes dans le métro. Il difflue sur son travail, son amie, leurs tentatives répétées d'avoir un enfant, son frère, son père, etc. Puis il se remémore deux rêves laissés jusque-là en latence. Dans le premier, il s'agit d'un barman; dans le second, de la ville où il est né, où il se retrouve derrière la clinique.

Je l'écoute en pensant à ses mots inauguraux, «retard", "heurté», «métro ». Pour un psychanalyste, une revendication pulsionnelle infantile s'y laisse aisément deviner à partir du double sens de chacun de ces mots. En même temps, un analyste sait qu'une telle revendication dans les rêves et les séances masque des aspects traumatiques sous-jacents; que l'interprétation de la revendication doit donc être un choix qui n'ignore pas cette fonction dissimulatrice.

Mon patient poursuit son mouvement régressif formel de séance en évoquant un patient qui pue, qui en fout ainsi plein la gueule des autres, façon de leur rentrer dedans; que ce matin il a lui aussi, et il se demande bien pourquoi, heurté deux personnes. La revendication pulsionnelle avance sur la voie régrédiente corporelle, sensuelle et sensorielle. Il est possible de dire aussi que la régression suit son cours à rebours.

Mon patient a un ton vif, légèrement agressif. La veille, il avait fini sa séance sur un «ils font tous chier », après avoir utilisé sans s'en rendre compte, toute une série de mots scatologiques évocateurs de la matière fécale. J'avais ponctué par un «c'est le cas de le dire» des plus énigmatiques, pour lui qui 
filait sa régression fécale bien logiquement sans réflexivité. En effet, en acceptant dans cette séquence la situation analytique, ce patient suivait la règle fondamentale et me déléguait l'écoute signifiante. Il interprétait associativement sa pulsion, et me laissait le soin d'interpréter le dévoilement de cette pulsion engagée dans son discours, ainsi que le sens de cette présentification à ce moment-là de son analyse. Le retour de la revendication peu accessible de la veille se présentait donc de façon plus nette lors de cette séance. Par contre mon intervention actualisait brutalement son propos en me proposant faisant partie des «ils».

Signalons encore que ces séances se situent à un retour de vacances. Ce qui s'y exprime est donc aussi un après-coup des vacances, un après-coup d'une vacance, donc une revendication sollicitée par la confrontation à cette vacance, revendication venant transformer un déplaisir en réalisation de plaisir.

Le fil rouge de ses associations apparaît et tient compte de ces aspects contextuels; de son identité d'analysant (régression formelle de séance, soustendue par une aspiration à une régression sensuelle liée aux revendications pulsionnelles); de la séance de la veille dominée par des figures de fécalité pulsionnelle; de la proximité des vacances qui viennent de se terminer; de la récurrence de ses associations sur ses tentatives d'avoir un enfant, etc. Seule la métapsychologie permet d'entendre un fil latent mêlant les temporalités, les souvenirs récents, passés, recomposés, et ceux de l'enfance.

Suivons ce fil de l'écoute et de l'entendement psychanalytique : retard (de règles), heurter (une revendication pulsionnelle sadique mais aussi choquante), métro: mettre-trop, barman : barre-man, clinique: cli-nique, puer, foutre, etc. La revendication anale homosexuelle s'impose de façon prédominante. Elle intègre en même temps la haine liée à l'exclusion de l'absence. Mais elle dit en même temps une revendication infantile sous-tendue par une théorie sexuelle infantile. L'interprétation devient possible: «c'est par un heurt anal que l'on fait les enfants; faites-le moi!»

Mon interprétation sera prise bien sûr pour de la semence. Sur le pas de la porte, il me dit, «merci »! Cette fois, je l'avais anticipé. Mais cela ne m'avait pas empêché de formuler l'interprétation, bien que je devinais qu'elle serait utilisée au service de la satisfaction de la revendication et non pas au service du renoncement.

Ce moment de clinique quotidienne a l'avantage de nous montrer qu'un transfert d'une revendication infantile sollicité présentement par le contexte de séance, sert de véhicule à un autre transfert plus refoulé. Se mêle donc à 
une réactualisation d'une revendication passée, une actualisation d'une revendication atemporelle qui tente de devenir intemporelle. Nous assistons là à un véritable travail de transfert de séance.

\section{Interprétation et après-coup}

L'interprétation de ce transfert d'émergence est une interprétation portant sur le premier temps du procès de l'après-coup, au service de l'enrichissement du préconscient et de la consolidation du narcissisme, une interprétation substitutive en faveur des réalisations hallucinatoires de désir, et non pas une interprétation résolutive au service du renoncement.

Toutefois, un premier renoncement a lieu dans ce cas aussi du fait de la représentance que constitue le maintien du langage exigé par la règle analytique. En effet, le langage ne permet pas une satisfaction du même type que celle obtenue par les images du rêve plus proches de l'informe de la pulsion, et encore moins par le biais d'une régression sensuelle telle que le permet l'érotisme qui traverse langage et figuration pour aller au-delà, vers une inscription des frayages corporels obtenus par conversion. Ces deux modalités d'interprétation articulent le travail de rêve et le travail de transfert.

\section{Les temporalités de l'après-coup}

Avec l'après-coup, c'est toute l'organisation temporelle du fonctionnement psychique que nous convoquons, sa dynamique certes, mais aussi sa fonction économique. Il permet aussi une inférence sur la structuration topique selon plusieurs instances articulées les unes aux autres par cette dynamique. Le procès de l'après-coup est la forme même du fonctionnement psychique.

L'après-coup évoque avec évidence la dimension temporelle, puisqu'il se réfère ouvertement à un espace temporel, une durée. Ce concept est donc consubstantiel à toute réflexion sur le temps dans la psyché.

Par sa discontinuité, il permet l'accès de la conscience au temps, et l'accès du temps à la conscience. La régression inaugurale de ce procès se fait jusqu'aux motions pulsionnelles définies par leur tendance au retour à un état antérieur, dynamique à l'origine du sentiment diurne d'être occupé par une tendance au disparaître. Au cours de ce processus se rencontrent les diverses temporalités impliquées dans la psyché de façon complémentaire mais aussi contradictoire; d'où la notion proposée par André Green de temps éclaté (Green, 2000). Nous retrouvons la classique intemporalité de l'inconscient, ce hors temps nécessaire au déni nocturne du temps, mais aussi la 
capacité de la psyché à remonter le temps, à se régénérer, rajeunissement par le bain de jouvence que constituent les rêves.

L'après-coup se retrouve donc occupé et déterminé par cette tendance au disparaître, mais aussi par cet autre, contraire, la tentative d'inscrire ce qui tend à disparaître (Camus, 1949; Clair, 1983, 76) ${ }^{1}$.

Soulignons encore que le terme courant d'après coup ne garde dans sa signification manifeste qu'une temporalité de successivité dessinant une durée, le trajet d'un passé et d'un avenir, voire d'un futur antérieur. L'aprèscoup c'est ce qui advient, ou qui est censé advenir, après qu'une autre chose antérieure ait eu lieu. La réalité de la tendance au disparaître, réalité dissimulée par le terme lui-même, revient plus clairement dans le substantif français coup, que dans le terme allemand. Le déni n'est pas aussi aisé que souhaité! Ce qui est mis dehors, par la porte, revient par la fenêtre.

En étant la forme même du fonctionnement psychique, l'après-coup est la forme implicitement portée par toutes les productions de la psyché. Elles sont toutes déterminées et grosses de l'histoire qui les a fondées. Elles transmettent ainsi toutes comme message, l'histoire et la temporalité de leur genèse.

\section{Le procès de l'après-coup et les formations de la pensée}

Notre cheminement précédent nous a appris que le terme d'après-coup désigne tout à la fois le procès du travail psychique et le résultat de ce dernier. Ce sont ces formations résultats de la mentalisation, ces produits de la pensée qui sont généralement désignés en tant qu'après-coups, laissant dans l'ombre le procès complexe de l'après-coup lui-même.

Parmi ces résultats, on retrouve au premier plan les symptômes, le rêve, la parole de séance c'est-à-dire la libre association (la parole d'incidence), mais aussi le transfert; en fait l'intégralité des productions de la psyché.

Cette importance de l'après-coup, en tant que forme du procès de la constitution de la pensée, se trouve corroborée par le fait qu'il est historiquement présent dès les prémices de la psychanalyse, voire même en tant que forme inchoative, préalable, mais aussi forme potentielle, en tant que matrice de la psychanalyse. Nous pourrions ainsi dire que la vie psychique est un après-coup d'une potentialité qui aurait pu ne jamais advenir; d'où cette autre conséquence complémentaire, l'après-coup installe et crée aussi bien le résultat manifeste que le cheminement inconscient. Donc l'inconscient est lui-même un après-coup; de même que l'objet qui est ni donné, ni déjà là. 
Psyché est un entre-deux terrestre, placé entre deux irreprésentables, le rien du disparaître, et l'inaccessible inscription de l'altérité de la perception.

La genèse biblique est elle-même construite ainsi. Le paradis est un aprèscoup de la différence des sexes. C'est le fait de déciller les yeux, l'éveil de ce qui a toujours existé mais qui était dénié en tant que différence traumatique, qui contraint la psyché à créer, après coup, le paradis perdu, lieu mythique qui n’a jamais existé. Mais la reconnaissance de la différence des sexes est aussi un après-coup. Elle a lieu après un temps de déni, certes; mais surtout elle a lieu suite à l'éveil de la tendance régressive au disparaître qui voit et reconnaît en la différence des sexes sa concrétisation. Elle a donc lieu suite au bris du déni. C'est ce que la clinique vient de nous apprendre, que l'après-coup utilise le mécanisme du déni pour se réaliser.

Nous voici en mesure de mieux cerner ce procès essentiel du fonctionnement psychique, l'organisation en deux temps de la sexualité humaine étant la plus grande preuve de cette dimension essentielle.

L'après-coup, c'est le procès rendu nécessaire par la tendance au disparaître, à laquelle doit répondre la tentative d'inscrire ce qui tend à disparâ̂tre, avons-nous déjà précisé. Ce qui tend à disparaître dans l'informe, c'est la pulsion; ce qui s'inscrit en de multiples formes, ce sont toutes les formations psychiques, reliées à la conscience par le biais du langage. Psyché se situe entre le disparaître de la pulsion et l'épitaphe du langage.

Autrement dit, l'après-coup est la forme du fonctionnement psychique qui tente d'articuler l'informe pulsionnel à la gravure langagière idéale, au mot sans son ni trace; et ceci par le biais de la production de formations psychiques et de diverses déformations et transformations du pulsionnel. Il s'agit donc d'un procès générique qui aboutit à des résultats multiples, constituant toute la kyrielle possible des formes cliniques.

Si ce procès apparait ainsi dans son universalité, il est utile de souligner qu'il n'est en aucune façon la garantie d'un accomplissement; il est d'abord et avant tout une tentative, référée à un aboutissement hypothétique et virtuelle. Du point de vue clinique, il existe de multiples avatars et de non moins nombreux achoppements à sa réalisation. Par exemple, nous connaissons bien les rêves de plusieurs nuits successives, occupés à élaborer par étapes une dimension traumatique qui ne peut se résoudre en une seule nuit, en une seule nuit de rêve.

Cette généralisation comme modèle du fonctionnement psychique n'enlève donc rien à la diversité en tant que résultats fondant la multitude des tableaux cliniques par lesquels se traduisent les achoppements que le procès 
peut subir au cours de sa réalisation. Comme nous le savons, ce sont ceux-ci qui rendent possible l'accès aux opérations qui fondent l'après-coup, alors qu'elles restent inaccessibles lorsque le fonctionnement se fait de façon optimal. Dans ce cas, le proverbe se trouve confirmé: à quelque chose malheur est bon!

\section{La tendance au disparaître et l'impératif d'inscription}

Nous avons déjà largement abordé comment l'après-coup est un procès qui conjugue une tendance au disparaître et un impératif d'inscription. Précisons encore que pour arriver à ses fins, il doit produire toutes les formations psychiques constituant la réalité psychique, tant celles régressives que celles accomplies. Tout l'espace psychique est créé ainsi. C'est ce qui permet de dire que tout désir mature est un après-coup accompli; mais aussi que les formations régressives et archaïques sont aussi des après-coups indispensables, constitutifs des voies intérieures, des voies de frayage et de régrédience.

Pour traiter la tendance traumatique au disparaître, le psychisme doit se donner une infinité de contenus régressifs composant les auto-érotismes. Ceci est évident avec le rêve. Pour traiter ce qui a valeur traumatique durant le jour, le rêve suit une voie régrédiente grâce à des matériaux régressifs qu'il fabrique au fur et à mesure de ses besoins. Ce temps de la régression et de l'intemporalité permet de répondre au hors-temps, à l'a-temporalité du traumatique, en faveur d'une transformation du pulsionnel traumatique en intemporalité, et de la génération d'une libido apte à se tourner vers le monde extérieur, vers les objets.

Sont donc impliquées les attractions régressives extinctives propres à la vie pulsionnelle, ainsi que l'impératif psychique à inscrire et à orienter l'économie libidinale sur la voie progrédiente, vers les matériaux d'inscription, la conscience et les objets du monde. Telle est la mission de ce procès constitutif de notre précieuse matière psychique.

Nous risquons toujours avec l'après-coup, le poids des mots y participe, de privilégier le sens de l'usage commun: quelque chose qui arrive après une autre antérieure; c'est-à-dire le sens du mot originaire allemand : porter vers l'avant. La mission rétroactive est alors perdue, la générativité aussi. Le lien entre les deux événements devient un simple lien de temporalité linéaire, ce que nous appelons en psychologie le point de vue génétique, avec l'idée d'une genèse orientée par la flèche du temps: passé, présent, avenir. Alors que ce que nous montre la psyché, c'est que ce procès travaille dans les deux sens, 
d'où le double sens de tous les contenus psychiques dès que nous abandonnons la stricte rigueur monosémique. Le double sens apparaît très fréquemment, par exemple dès que nous plaisantons, mais aussi dans le discours amoureux, et en séance bien sûr, quand un patient s'abandonne à la libre association et un analyste à l'écoute régrédiente. Le discours de séance peut alors prendre une tonalité comique, excepté lorsque la dimension traumatique se révèle très prégnante, empêchant tout double sens sexuel.

L'analyste est censé connaître la fonction essentielle de ce double sens, et ne pas se laisser séduire par lui, c'est-à-dire détourner et distraire de sa propre fonction. Il est tenu à une double mission. Il se doit de soutenir le double sens de la réalité psychique et ne pas succomber aux sirènes du traumatique en devenant un psychothérapeute réparateur, privant alors la psyché de ce qu'elle a de plus spécifique: sa capacité par le fantasme d'échapper par moments, aux rigueurs de la réalité du renoncement. On se souvient des premiers vers du Faust de Goethe: «Venez illusions !.... au matin de ma vie» etc. (Goethe, 1877).

Mais il se doit aussi de ne pas utiliser ce double sens du fantasmatique au service du déni de traumatique.

\section{Les activités psychiques régressives de la passivité et l'intemporalité de l'inconscient}

Comme nous l'avons déjà évoqué, le modèle de l'après-coup fut préconçu avant même son élaboration au sein du corpus théorique de la psychanalyse, par le modèle de Charcot des deux temps des symptômes hystériques. En effet, l'agencement en deux temps, de l'événement puis du résultat symptomatique, temps manifestes qui inaugurent et concluent le procès, avait déjà été décrit par Charcot à propos de la constitution des symptômes, avec un premier temps, celui du trauma, du choc, l'événement traumatique, et un deuxième temps, celui du symptôme, de la production, la formation symptomatique. Si Charcot en était resté à une logique temporelle linéaire et chronologique, il avait néanmoins désigné et nommé le temps intermédiaire, temps d'élaboration psychique, qui deviendra sous la plume de Freud, via la rétrogression de Breuer, le temps de latence.

La part de vérité psychique la plus importante de la conception du Maître de la Salpêtrière ne réside pas en cette observation des deux temps, encore moins dans la reconnaissance d'une étiologie liée à un trauma-choc événementiel, mais dans le fait d'avoir dénommé l'entre-deux-temps, incubation psychique, élaboration psychique, et de ne pas l'avoir inscrit en tant que 
troisième temps d'un processus en trois temps manifestes, en décalant cet entre-deux-temps de la numération. Il a agi ainsi la position de l'entre-deuxtemps, d'être en latence des deux autres. Il a donc eu l'intuition de la mise en latence, et du travail de la latence.

Par contre, c'est Breuer qui découvre qu'il est possible de remonter à rebours les deux temps linéaires jusqu'au moment traumatique. Il appellera ce phénomène la rétrogression, et la méthode thérapeutique qui l'utilise, la catharsis.

Étayé sur ces prémisses, et occupé par sa quête de l'étiologie, Freud explorera à rebours la généalogie du symptôme, puis de l'ensemble des productions psychiques, en particulier les formations de l'inconscient, le rêve en étant le prototype. En suivant cette logique étiologique du symptôme (ses neurotica), Freud explore la causalité et la temporalité des productions psychiques. En voulant préciser leur détermination, il découvre la surdétermination et l'intemporalité qui y préside.

C'est donc en prenant très au sérieux l'observation empirique temporalisée de Charcot, en en faisant l'un de ses points de départ, que Freud découvre un registre psychique qui participe au travail psychique, et qui échappe à la temporalité. L'autre remarque clinique à laquelle Freud accorde toute son attention, c'est l'observation faite par Breuer de la disposition d'Anna O. à la remémoration spontanée, et de l'effet thérapeutique de cet acte de mémoire, acte de retour à rebours dans le temps. Il reconnaît alors l'autre versant, conservateur, de cette intemporalité. L'inconscient va trouver ses deux faces, pathogène par sa dimension posthume, et anti-traumatique par la conservation qu'il assure. L'intemporalité est une nouvelle catégorie, à la valence double.

Freud ajoute alors la régression au processus de production d'un symptôme manifeste en deux temps. Il découvre que le passé s'actualise dans le présent, et qu'il n'est donc pas un véritable passé. Il est en mesure de compléter la théorie des productions expressives, par l'ajout de ce temps de régression liée à l'attraction négative traumatique, et d'un temps de travail spécifique dans le champ régressif, un travail de la régrédience. Freud définit le rêve en tant que produit psychique régressif.

Cette investigation du régressif lui permet de tenter une intelligibilité de l'ensemble des formations de l'inconscient, l'ensemble des activités psychiques régressives de la passivité, le rêve étant leur prototype. Cette intelligibilité s'applique aux symptômes, mais aussi à la scène analytique, à la parole de séance, cette parole d'incidence, à l'écoute de l'analyste, son attention en égal suspens, mais aussi à la rêverie diurne, à la créativité artistique, à la scène érotique et 
la vie sexuelle, etc. Elles sont toutes rendues possibles par l'intemporalité de l'inconscient.

\section{Le cas Emma et la double temporalité inversée}

Utilisons maintenant, pour illustrer les développements précédents, quelques vignettes et évocations cliniques extraites de l'œuvre de Freud. Elles se situent dans une dynamique historique, et font désormais partie de l'histoire, de notre culture. Si nous incluons à l'œuvre publiée les quelques 20 à 30000 lettres composant la correspondance (Molnar, 2007), il est possible de trouver de fréquentes allusions à d'autres situations cliniques que celles publiées par Freud.

C'est dans les Lettres à Fliess (Freud, 1950c [1895], 307-396) que Freud présente le cas Emma et décrit ce processus en deux temps, et son exploration à rebours. En 1895, année des Études sur l'hystérie, Freud explicite plus avant le phénomène de l'en-deux-temps et de l'entre-deux-temps dans le chapitre «Proton pseudos» de son Projet de psychologie où il expose le cas d'une jeune femme qu'il dénomme Emma.

Emma présente une agoraphobie des boutiques. Son symptôme se corrèle avec deux souvenirs inconscients renvoyant chacun à une scène; une récente, celle des commis, et une autre plus ancienne, celle de l'épicier. Entre les deux, la puberté d'Emma. Freud remplace ainsi le choc événementiel de Charcot par un événement choquant, une séduction transgressive ayant valeur d'éveil trop précoce de la sexualité. La reconstitution de la genèse du symptôme fait de la scène ancienne, la scène 1 , et de celle récente, la scène 2 . Une nouvelle conception du symptôme apparaît, en tant que réminiscence.

Mais la voie clinique qui permet cette élaboration temporelle de l'étiologie, exige d'inverser la temporalité, inversion qui s'accompagne d'un espoir thérapeutique basé sur la remémoration à-rebours, chaque souvenir devenant un après-coup d'un souvenir inconscient ayant acquis, dans l'après-coup de son refoulement, la valeur de coup traumatique. D'où la lente mise au point d'une nouvelle méthode et la différentiation des modalités de parole et de pensée qui la constitue, la libre association (Emmy v. N. ; Lucie R.), la règle fondamentale (Elisabeth v. R.) (Freud, 1895, 81, 127-132, 165, 174-175), puis plus tard, l'attention en égal suspens.

\section{Les sens dessus-dessous de l'après-coup.}

Dans ce contexte théorique, les symptômes acquièrent la valeur de formations posthumes, le refoulement ayant valeur de mise à mort. En 
découlent dans la théorie une série de notions telles que le refoulement, la résistance, les retours du refoulé, les retours de souvenirs posthumes, particulièrement ceux sexuels, sous la forme de symptômes substitutifs. L'idée d'un travail sous-jacent, propre à la période de l'entre-deux temps, en découle aussi; un travail de substitution. La doctrine du rêve exaltera ces notions et proposera le modèle du travail de rêve.

Freud ajoute à sa méthode ce qu'il a repéré depuis l'hypnose et qui lui a fait abandonner celle-ci, l'existence d'une résistance, d'où l'introduction, dès 1895, de la Règle Fondamentale, règle venant remplacer l'effort fait par le thérapeute hypnotiseur pour vaincre les résistances du patient, effort s'avérant être une résistance du médecin à la passivité.

C'est alors, voulant rehausser cette conception de l'en-deux-temps de la formation des symptômes et insister sur les procès sous-jacents à cet entre-deux-temps, que Freud crée en 1897, dans sa lettre à Fliess du 14 Novembre, le néologisme féminin Nachträglichkeit à partir de l'adjectif et adverbe courant Nachträglich, "porter après », porter vers l'après; " porter en avant ».

L'avènement de la Règle est donc une première impersonnalisation de la contrainte soutenue auparavant par l'hypnotiseur puis par le médecin. Elle remplace progressivement l'influence antérieurement obtenue par un forçage actif et par la prescription de souvenir. De ces techniques pré-analytiques sont nées celles proto-analytiques telles que l'associativité dirigée, encore à l'œuvre dans la Traumdeutung, lors de l'analyse du rêve de l'injection faite à Irma, reprise ensuite par la méthode du Rêve Eveillé Dirigé. Mais si la règle s'oppose à l'attraction régressive de l'atemporalité, elle invite aussi à un abandon à la passivité régressive. Elle exige donc, via l'intemporalité, l'inscription temporelle des produits régressifs. Paradoxale, elle prescrit le sens dessus-dessous.

La contrainte de la règle traduit un impératif de verbalisation en séance, rejoignant l'impératif d'inscription déjà évoqué, ce dernier ayant pour finalité la présentification sur l'écran interne de la conscience. En séance, il s'agit d'inscrire par une énonciation temporalisée, sur l'écran de la conscience tant de l'analysant que de l'analyste.

\section{Retour à Emma. Les temps 1 et 2 ; les scènes I et II.}

Revenons à Emma. Freud y décrit en fait trois événements rétrogrades du point de vue temporel, séparés par deux temps de latence. C'est la production manifeste du symptôme, la phobie d'entrer dans un magasin, qui se présente 
en premier au clinicien. D'après la conception de Charcot, il s'agit du temps 2 de la clinique symptomatique, le temps 2 de la genèse du symptôme, à partir duquel, d'après Breuer, il convient de rechercher le temps 1 du choc traumatique, le futur choquant régressif de Freud.

En suivant la méthode de la remémoration, le thérapeute peut se lancer à la recherche de ce temps 1 qui est en fait un temps de remémoration de séance. Freud nomme cette première remémoration la scène I. Pour Emma, il s'agit de la rencontre, justement dans un magasin, de deux commis aux sourires énigmatiques et évocateurs, pensées qu'Emma ne peut préciser. Ces sourires ont une tonalité clairement sexuelle. Ils sont séducteurs et persécutoires pour Emma qui doit les fuir afin d'éviter une constellation angoissante. Elle développe une phobie des boutiques.

Mais Freud ne se satisfait pas de ces deux premiers temps et de cette seule scène, celui du symptôme et celui de la remémoration aisée d'un événement conscient récent déclaré déplaisant, la rencontre des commis. Riche de l'expérience de Breuer, avec le cas Anna O., il poursuit son investigation. En effet, Breuer, suite à Charcot avec l'hypnose, et Bernheim avec la suggestion, cherche à explorer la remémoration de sa patiente jusqu'à un événement traumatique oublié, conçu comme le noyau traumatique impliqué dans la genèse même du symptôme, le véritable temps 1 . Rappelons qu'il s'agissait pour Breuer de réaliser une catharsis en suivant le processus de la rétrogression, c'est-à-dire la remémoration et les retrouvailles de l'événement traumatique, puis la reprise du développement à partir de cette remémoration, réécriture donc de l'histoire d'une façon différente, libérée de la valence traumatique de l'événement pathogène.

L'investigation de Freud se poursuit alors au-delà de la scène I, celle des commis pour Emma. Ne pouvant se satisfaire d'un souvenir aussi facilement remémorable, il insiste auprès d'Emma qui lui fournit un autre souvenir beaucoup plus ancien, à peine remémorable, une scène II bien plus ancienne, refoulée au sens propre du terme, celle de sa visite, petite fille, dans une boutique tenue par un épicier, ce dernier lui ayant posé la main sur le bas-ventre à travers ses vêtements. Emma précise qu'elle est retournée dans la boutique de l'épicier une seconde fois.

Freud considère que l'événement traumatique déterminant, donc le temps 1 de la genèse du symptôme, est cette scène II de séance. Ce temps 1 selon la chronologie reconstruite, correspond en séance à la scène II, puisqu'elle est remémorée en second. Cette scène n'a donné lieu à aucune différenciation en termes de représentation et d'économie libidinale. Elle 
constitue le temps 1, ce temps précoce, prématuré pour l'intégration des motions pulsionnelles sollicitées lors de cette situation.

Les qualités temporelles, précocité et prématurité, trouvent dès lors leur place dans les logiques métapsychologiques de Freud qui peut poursuivre à nouveau sa théorisation. Il envisage que l'événement-trauma date de l'enfance, qu'il est inconscient par refoulement, et que sa valeur traumatique dépend de sa nature posthume. Il s'agit pour lui, comme pour Breuer, de débloquer les affects dits coincés, de réaliser une catharsis, mais surtout d'obtenir une meilleure liaison des contenus inconscients à la conscience par le biais des représentations de mot du processus secondaire, liaison s'accompagnant d'une modification radicale de l'économie de l'événement traumatique en cause. Cette meilleure liaison, il la relie au statut psychique des mots, l'efficience de ceux-ci étant plus grande quand ceux utilisés sont en adéquation avec les motions et pensées refoulées. Telle est le rôle de l'interprétation, d'apporter, tant par le don de parole que par ses contenus, un surinvestissement qui relie l'économie régressive à la conscience, d'où la possibilité d'orienter les investissements libidinaux vers les objets.

Freud abandonnera la notion de rétrogression au profit de celle d'une régression permettant d'accéder à de telles modifications et transformations portant sur l'économie par le biais des contenus verbaux. Ainsi libérée de cette fixation traumatique, la psyché pourra produire de nouvelles formations de pensées non frappées par cette détermination traumatique.

La notion d'après-coup apparaît dans cette logique recouvrir tout à la fois la formation de symptôme, en tant qu'après-coup du temps traumatique, mais aussi l'avènement d'une nouvelle modalité d'un penser libéré, qui s'avère être également un après-coup du traitement du noyau traumatique, mais un après-coup qui a atteint son plein accomplissement.

\section{Le diphasisme de la sexualité humaine et la résolution œdipienne}

Freud se rend compte très tôt au décours de sa recherche étiologique, que la sexualité est concernée. Il envisage une étiologie sexuelle, une séduction sexuelle précoce, puis une sexualité qui ne respecte pas les lois de la psyché et qui est prise dans des visées transgressives, en particulier incestueuses. Les symptômes sont des expressions sexuelles anachroniques. Freud perçoit que cette généralisation au fonctionnement psychique trouve en la sexualité sa démonstration. En effet, la sexualité humaine est construite sur ce modèle du en-deux-temps avec une longue période de latence entre-deux. Le diphasisme 
de la sexualité s'avère être le prototype de toutes les productions psychiques. Ainsi la sexualité adulte postpubertaire est-elle un après-coup de la sexualité infantile, avec la période de latence comme entre-temps; de même la sexualité infantile est un après-coup du pulsionnel traumatique, rendu possible par la résolution œdipienne et l'installation de l'inconscient intemporel. Pour le dire directement, les préliminaires de l'érotisme ne le deviennent que d'avoir subi un endeuillement œdipien; sinon ils seraient organisés en perversion monophasique. La perversion polymorphe de l'infantile n'existe que sous couvert d'un renoncement œdipien. Les troubles de la sexualité de l'adulte sont déterminés par les perturbations de cette résolution, perturbation empêchant aussi la sexualité infantile inconsciente de fonctionner selon des auto-érotismes psychiques. Le défaut de refoulement ne laisse pas apparaître la sexualité infantile, mais une pulsionnalité qui devra être maitrisée par des systèmes défensifs divers, les perversions en particulier mais aussi bien d'autres.

Ce qui s'avère être traumatique, et Freud l'exprimera clairement en 1925, c'est la sexualité infantile quand elle est insuffisamment contre-investie et circonscrite dans l'inconscient, donc quand le renoncement aux objets oedipiens ne s'est pas soldé par l'avènement d'un surmoi contre-investissant, ce dernier étant censé permettre un destin érotique objectal au sexuel infantile.

\section{La cooptation d'un événement récent pour le rêve de la nuit suivante}

Cet intérêt pour le symptôme, et en particulier les symptômes hystériques et névrotiques, amène Freud à faire un autre détour afin de théoriser le travail psychique de l'entre-deux-temps. Il s'intéresse alors au rêve et élabore sa doctrine du rêve. Cette formation nocturne implique les souvenirs tant de la veille que ceux anciens de l'enfance, c'est-à-dire proche d'un sexuel pulsionnel. Elle mêle donc plusieurs temporalités. S'y dessine un doublet mnésique constitué de souvenirs récents et de souvenirs beaucoup plus anciens, le souvenir récent étant de moindre intensité traumatique que le souvenir refoulé.

Cet autre point retient encore l'attention de Freud, cette articulation des souvenirs, des temporalités et des économies. Il perçoit que les commis d'Emma sont eux-mêmes déterminés par l'épicier refoulé. Pour le dire autrement, Emma a besoin de chercher-trouver-créer des commis afin de pouvoir traiter cette expérience trop précoce d'avoir été sollicitée sexuellement par l'épicier. En fait, que cela ait eu lieu ou non, c'est la vérité de la psyché d'Emma qui importe. Ainsi Emma n'a pas été traumatisée par les commis, 
elle est allée chercher-trouver des commis pour pouvoir modifier l'économie sexuelle de l'expérience traumatique épicier.

C'est cette logique de pluridétermination que Freud retrouve dans le rêve en la compliquant encore. Les sources déterminantes des formations psychiques de l'inconscient intègrent certes les souvenirs récents et ceux anciens reliés les uns aux autres de façon analogique et symbolique, mais aussi d'autres facteurs. Freud en décrit deux autres : les sources somatiques, donc l'influence des sensations proprioceptives du corps somatique, et la typicité c'est-à-dire les lois du fonctionnement psychique lui-même, les ressentis liés aux degrés de réussite et d'échec du travail psychique. Freud aborde ce dernier point par le biais des rêves typiques et des affects typiques que sont la honte, la culpabilité, et la douleur morale.

Les rêves et les symptômes sont déterminés par ces sources et par les opérations propres au processus primaire, la condensation, le déplacement et la figurabilité, c'est-à-dire le travail de déformation des contenus verbaux en images-rébus, opérations auxquelles il convient de rajouter, surtout pour les symptômes diurnes, leur élaboration secondaire, c'est-à-dire ce surplus de déguisement permettant de présenter le résultat final à la conscience.

Après ce détour par le rêve et sa dynamique en deux temps se déroulant dans les deux directions de la pensée, Freud peut aborder le fait que le récit du rêve est également un après-coup prolongeant le rêve lui-même, qui fut lui-même un après-coup pris entre les attractions régressives traumatiques et l'impératif à produire un matériau psychique.

Avec ce détour par le rêve se précise un point important. Les pensées mises en latence le sont parce que le rêve en a besoin pour réaliser ses fonctions. La mise en latence n'est donc pas mue seulement par le processus secondaire; au contraire, l'attraction régressive intervient tout particulièrement dans la cooptation de certaines pensées diurnes. Le travail de rêve se prépare par cette cooptation diurne. Le rôle des séances devient plus clair. Elles potentialisent ce mécanisme, voire même s'offrent comme matériau à coopter.

\section{Un troisième après-coup, le transfert de séance}

C'est avec l'Homme aux loups que Freud envisage que les rêves de séance, mais aussi l'ensemble du discours de séance, et le transfert lui-même, sont à penser selon les mêmes logiques de l'après-coup. Il fait alors de la séance un troisième après-coup, après le symptôme et le rêve. Ainsi rêve, symptôme, transfert se retrouveront-ils dans la même lignée de la tentative d'une psyché 
d'atteindre son accomplissement, par le biais de l'installation et de l'efficience du procès de l'après-coup.

À partir de 1917, l'usage du concept d'après-coup est abandonné par Freud, au profit de l'inscription dans la théorie du diphasisme de la sexualité humaine et des deux temps du complexe de castration. S'y ajoute l'implication, dans l'agencement en deux temps de tout travail psychique, de la tendance régressive, tendance au retour à un état antérieur propre à la dualité pulsionnelle, puis l'impératif surmoïque faisant contrepoint à cette tendance. Il est probable que cet abandon est lié à la reconnaissance progressive par Freud de l'importance du travail régressif masqué par la lettre du terme d'après-coup.

\section{L'oubli et le déni, un chiasme des temporalités}

Tournons-nous maintenant vers un autre exemple, extrait de la clinique du processus de théorisation de Freud. Ce dernier nous livre un symptôme personnel banal de la vie quotidienne, l'oubli d'un nom, celui de Signorelli. En 1898 (Freud, 1898, 241-251), Freud étudie avec détails et précision la concaténation associative à rebours lui permettant de remonter de l'oubli jusqu'au mot de Signorelli, en passant par d'autres noms de peintres, de lieux, etc. Nous connaissons bien ce long cheminement présenté selon un véritable circuit de ramifications, celui des associations à rebours passant par Botticelli et Boltraffio, et aboutissant au jugement dernier, donc à une culpabilité inconsciente.

Puis en 1901, dans le premier chapitre de la Psychopathologie de la vie quotidienne (Freud, 1901, 79-85), Freud reprend la question de l'oubli des noms propres, et revient sur le cas de Signorelli. Il nous apprend alors qu'au moment de son symptôme, il était sous le coup, le choc d'une annonce traumatique, la mort d'un ancien patient turc ayant choisi de se suicider après l'annonce d'une impuissance médicale incurable. La dimension traumatique se trouve donc éveillée, et renvoie à des éléments traumatiques d'enfance, la mort du frère de Freud, Julius, par exemple. Mais ce qui est à remarquer ici, c'est que l'accès à l'associativité et à la concaténation permettant de faire ce cheminement à rebours dans le champ de la régrédience s'est déroulé sous couvert d'un déni temporaire nécessaire à cette activité psychique régressive et passive de la libre association. Nous retrouvons à nouveau le modèle du rêve et du déni installé au cours de l'endormissement afin que le rêve puisse prendre place.

C'est seulement dans un second temps, après un long déroulement dans l'espace régressif, que Freud peut, comme le rêveur après son réveil, réintégrer 
la dimension traumatique diurne, corrélée avec celle de son enfance (disparition de son frère en particulier), et au-delà avec la tendance pulsionnelle au disparaître, la régressivité pulsionnelle. Il peut alors nous offrir sans le théoriser lui-même, un bel exemple des deux temps à rebours du traitement de l'âme, le frayage régressif premier se faisant sous couvert du déni de ce qui fut initiateur du trouble, le moment de vécu traumatique.

\section{Les deux temps de la théorisation}

Soulignons qu'il agit dans le même temps une élaboration métapsychologique en deux temps. La théorisation suit donc elle aussi le même modèle que la théorie de la formation des symptômes et des rêves, et que la théorie de la méthode de traitement. Ce modèle commun est celui de l'après-coup. L'interprétation se présente également prise dans cette même dynamique, avec, comme nous l'avons déjà abordé, un temps de participation substitutive, puis un second temps résolutif. Pour compliquer encore un peu, signalons que l'analyste ne sait lui-même qu'après coup si son interprétation était du premier ou du second type. En réalité, elle participe systématiquement des deux.

Une question se pose évidemment envers les patients, ou plutôt les moments de cure où un patient n'arrive pas à réaliser cette mise en latence, occupé qu'il est préférentiellement par une névrose traumatique actuelle. Comme Freud, nous assistons alors à une tentative de réaliser l'après-coup, tentative répétitive qui avorte tout aussi répétitivement.

\section{Le temps du déni, le temps de sa révélation}

Un autre exemple va nous aider à illustrer cette dualité de l'interprétation. Toute interprétation est prise dans deux logiques : l'une qui tient compte du contexte associatif incluant la séance, l'analyse, l'analyste, le tableau symptomatique, etc., et l'autre qui est fondée sur ce que l'analyste ignore, c'est-àdire sur ce qu'il dénie alors qu'il s'agit d'une méconnaissance. En séance, toute méconnaissance a valeur après coup de déni. L'analyste est censé savoir. Toute interprétation est prise dans ce double sens, de tenir compte de certaines réalités du contexte, et d'en dénier d'autres. Ainsi toute interprétation se modifie-t-elle au fur et à mesure que nous élargissons le contexte associatif d'un rêve, et change de sens selon qu'elle s'adresse au déni ou au désir inconscient dissimulé.

L'exemple suivant est encore extrait de l'œuvre de Freud. Il concerne un rêve célèbre, le rêve de la table d'hôte, rêve principal du premier ouvrage de 
vulgarisation que Freud a rédigé, Uber Traum (Freud, 1901). Ce rêve porte sur les rapports de Freud à sa propre femme, Martha.

Le rêve: «En compagnie, table ou table d'hôte... On a mangé des épinards... Madame E.L. est assise à côté de moi, se tourne régulièrement vers moi et pose familièrement la main sur mon genou. J'éloigne la main d'un geste défensif. Elle dit alors: Pourtant vous avez toujours eu de si beaux yeux... Je vois alors sans netteté quelque chose comme des yeux dessinés sur un dessin ou comme le contour d'une paire de lunettes...».

L'interprétation immédiate est que Freud a des visées sur une autre femme, le souhait d'avoir une aventure. Présenter sa femme infidèle, comme il le fait dans ses associations, l'autorise donc pleinement à poursuivre ses propres souhaits. Il invente la bergère afin de disposer de la réponse du berger à la bergère.

Cette interprétation est une lecture quasi directe du contenu manifeste négligeant le travail de déformation. La régression associative n'inclut alors pas l'enfance.

Freud se souvient d'une autre scène, quasi identique à celle du rêve, qui s'est déroulée entre lui et Martha au début de leur relation. Autre interprétation, cette fois nostalgique: ah le bon vieux temps révolu de l'état amoureux, de l'exclusivité!

Il reprend la même scène en 1901 dans Psychopathologie de la vie quotidienne (Freud, 1901, 225-226), et évoque l'oubli systématique des détails concernant Martha. Il rattache ce fait aux «égards » qu'il a pour elle. Lui aussi est donc « réservé ».

La correspondance de Freud avec Martha nous livre d'autres ouvertures. S'ensuit un élargissement du contexte de ce rêve. Lors d'une cure, les lettres sont remplacées par les séances successives avec la différence essentielle qu'elles arrivent avec d'autres rêves.

Nous apprenons alors que peu avant le rêve, Freud et Martha ont traversé durant leurs fiançailles la plus grande querelle qu'ils ont connue depuis le début de leur relation. Le différend susceptible de les amener à consommer leur rupture, porte sur une histoire d'argent avec le frère de Martha, personnage que Freud qualifie d'escroc. Freud est inquiet à propos de la dot de Martha qui est entre les mains de son futur beau-frère. Martha prend parti pour son frère, contre son fiancé.

Le rêve réalise donc le désir d'avoir une femme qui le confronte à une jalousie objectale, alors que dans cette histoire Martha le prive de celle-ci au profit d'une rivalité œedipienne, via ses amours d'enfance. Mais ce qui fait 
rêver Freud ce sont, bien sûr, ses propres désirs régressifs d'enfant éveillés par cette scène, et délégués à Martha. Lui qui est en train de découvrir sa constellation œedipienne, reproche à Martha de ne pas avoir renoncé à ses objets œdipiens, en fait d'éveiller ses propres aspirations transgressives.

Cet exemple nous confirme ce que nous avions abordé avec celui de Signorelli, que l'interprétation psychanalytique se fait en deux temps, le temps du cheminement régressif, puis celui de la levée du déni. Mais s'y ajoute un point essentiel, le lien entre le déni d'une réalité de perception et le contreinvestissement de la régressivité extinctive. L'interprétation est donc messagère de réalités intrapsychiques, en particulier de l'intemporalité de l'inconscient et des discontinuité temporelles; mais aussi de la tendance traumatique au disparaître, donc de l'atemporalité traumatique. Toute interprétation participe ainsi à deux logiques, celle de l'inscription, qui tend à s'étendre à l'infini tout en butant sur l'arrêt qu'elle agit, et celle contraire, de la tendance à échapper à toute énonciation, donc à disparaître, à s'éteindre.

L'après-coup transmet cet arrière-fond du disparaître. Il l'illustre par les points de fuite, les mises en abyme, les ombilics du rêve, de l'interprétation, de la théorisation, ces ombilics ouverts sur un au-delà de toute temporalité.

\author{
Bernard Chervet \\ 16, rue Jacques Callot \\ 75006 Paris \\ 39, rue Professeur Florence \\ 69003 Lyon \\ bernard@chervet.fr
}

\title{
Note
}

1. Cette expression rappelle celle de Camus à propos de la peinture de Balthus: «la tentative de maintenir à jamais ce qui disparaît déjà ", phrase reprise par Jean Clair dans le catalogue de l'exposition Balthus de 1983 à Beaubourg.

\section{Références}

Camus, A., 1949, Préface du Catalogue de l'exposition Balthus, New York, Pierre Matisse Gallery. Chervet, B., 2008, L'après-coup, la trace manquante et ses mises en abyme, Bulletin de la SPP, 90, 99-196.

Chervet, B., 2009, L'après-coup : la tentative d'inscrire ce qui tend à disparaître, Revue française de psychanalyse, 73, 5, 1361-1441.

Clair, J., 1983, Catalogue de l'exposition Balthus, Paris, Musée National d'Art Moderne, 76.

Freud, S., 1895d [1993-95], Études sur l'hystérie, OCF. P, II, Paris, Presses universitaires de France, 9-332. 
Freud, S., 1898b, Sur le mécanisme psychique de l'oubliance, in OCF. P, III, Paris, Presses Universitaires de France, 1989, 233-251.

Freud, S., 1901a [1900], Sur le rêve, OCF. P, V, Paris, Presses universitaires de France, 2012, 1571.

Freud, S., 1901b, Sur la psychopathologie de la vie quotidienne, in OCF. P, V, Paris, Presses universitaires de France, 2012, 73-376.

Freud, S., 1950c [1895], Esquisse d'une psychologie scientifique, in Freud, S, La naissance de la psychanalyse, Paris, Presses universitaires de France, 1956, 307-396.

Green, A., 2000, Le temps éclaté, Paris, Ed. Minuit.

Lacan, J., 1966, Écrits, Paris, Seuil.

Lacan, J., 1966, Le temps logique et l'assertion de certitude anticipée, in Lacan, J., Écrits, Paris, Seuil, 197-213.

Molnar, M., 2007 État présent des correspondances freudiennes, in Michaud, S. (dir.), Correspondances de Freud, Paris, Presse de la Sorbonne Nouvelle, 17-30. 